\title{
Marchiafava-Bignami disease:Two cases with magnetic resonance imaging and positron emission tomography scan findings
}

\author{
A. Nalini, Jerry M. E. Kovoor ${ }^{1}$, Rose Dawn ${ }^{1}$, Kumar G. Kallur ${ }^{2}$ \\ Departments of Neurology, ${ }^{1}$ Neuroimaging and Interventional Radiology, National Institute of Mental Health and Neuro Sciences, \\ ${ }^{2}$ Health Care Global, Bangalore, India
}

\author{
Address for correspondence: \\ Dr. A. Nalini, \\ Neuroscience Faculty Block, \\ National Institute of Mental Health \\ and Neurosciences, Hosur Road, \\ Bangalore - 560 029, India. \\ E-mail: atchayaramnalini@yahoo. \\ co.in
}

PMID: 19934569

DOI: $10.4103 / 0028-3886.57813$

\begin{abstract}
We report two patients with Marchiafava-Bignami disease (MBD). A 38-year-old male with chronic alcohol abuse developed acute onset cerebellar ataxia and altered sensorium. He was diagnosed to have acute form (Type II) of MBD. Magnetic resonance imaging (MRI) showed extensive lesions involving the corpus callosum in its entire extent and also bilateral corona radiata and centrum semiovale. Corpus callosum had heterogeneous signal changes with ring enhancement. Positron emission tomography scan demonstrated reduced cerebral glucose metabolism diffusely over both the cerebral hemispheres. The second patient was 55-year-old male with chronic alcohol intake developed acute onset vomiting followed by behavioral abnormalities and altered sensorium. MRI showed diffuse lesion involving entire corpus callosum with suggestion of necrosis. Both the patients subsequently recovered, the first patient is back to his previous occupation and the second patient could be rehabilitated with some lighter work in his previous work place. Functional brain imaging may help to understand the pathogenesis of acute MBD and possibly the behavioral manifestations.
\end{abstract}

Key words: Computer tomography scan, Marchiafava-Bignami disease, magnetic resonance imaging, magnetic resonance spectroscopy, positron emission tomography

\section{Introduction}

Marchiafava-Bignami disease (MBD) is a progressive neurological disease characterized by corpus callosum (CC) demyelination and necrosis and subsequent atrophy. The lesions can spread to the hemispheric white matter. ${ }^{[1]}$ The poor outcome in MBD has been attributed to the extracallosal involvement, however cases with good outcome have also been reported..$^{[2-4]}$ Genu of the $\mathrm{CC}$ is more frequently involved. Necrosis results in cystic lesions, mainly in the genu and splenium. ${ }^{[5]}$ Occasionally other structures such as optic chiasm and tracts, putamen, anterior commisure, cerebellar peduncles and rarely cortical grey matter (laminar necrosis), and ' $U$ ' fibres may be involved. ${ }^{[6]}$ The pathogenesis of MBD is unclear; a toxic factor present in cheap red wine has been implicated, the nature of which had not been yet identified. MBD may present in various clinical forms. Clinical features of acute MBD include seizures, impaired consciousness, rapid deterioration and death. The features of subacute MBD include variable degrees of mental confusion, dysarthria, behavioural abnormalities, memory deficits, interhemispheric disconnection states and impaired gait. ${ }^{[6,7]}$ Chronic MBD is less common and presents as mild dementia which progresses over a period of several years. ${ }^{77,8]}$ As the clinical features are nonspecific, computer tomography (CT) and magnetic resonance imaging (MRI) are essential to confirm the diagnosis. In CT brain CC appears hypodense and in the presence of hemorrhagic lesions hyper or isodense. ${ }^{[5,9]}$ There are only few reports on functional imaging, positron emission tomography (PET) and single photon 
emission computed tomography (SPECT) in MBD. ${ }^{[10,11]}$ We report two patients with acute severe form of MBD and the imaging findings on CT, MRI and PET-CT scan.

\section{Case Reports}

\section{Case 1}

A 38-year-old male was brought in 2008 with history of chronic alcohol abuse, brandy or country liquor consumption [arrack - distilled spirit $(90 \mathrm{ml}$ has 3 units of alcohol)] of minimum $180 \mathrm{ml}$ per day, for the last ten years. There was binge drinking in the last four years before admission. He developed acute onset cerebellar ataxia and in the next 12 hours lapsed into altered mental state. Neurological evaluation revealed the patient in altered mental state. Pupillary reactions were symmetrical. There were no lateralizing deficits and no signs of meningeal irritation. Vital signs were normal. Laboratory test showed a normal hemogram, liver and renal function tests, and serum electrolytes. Lumbar cerebrospinal fluid (CSF) showed no cells, protein of $62 \mathrm{mg} / \mathrm{dl}$ and glucose $73 \mathrm{mg} / \mathrm{dl}$. Bacterial and fungal cultures were negative. CSF VDRL was nonreactive. Serum retroviral status was negative. Ethanol level or serum thiamine levels were not estimated. EEG showed diffuse slowing with abundant theta activity.

Non-contrast-CT (NCCT) scan brain at admission showed diffuse hypodensity involving the $\mathrm{CC}$ in its entire extent, more in the genu. There were patchy hypodensity lesions in the periventricular white matter bilaterally [Figure 1a]. Contrast CT (CCT) showed no enhancement. Follow-up CT scan done 15 days later showed hyperdensity lesions in CC and decrease in periventricular hypodensity. MRI done after 13 days of admission showed heterogeneous signal changes in the CC. On T1-weighted images the lesions had a hypointense center with hyperintense periphery involving the $\mathrm{CC}$ in its entire extent and also involving bilateral coronal radiata and centrum semiovale. Lesions were hyperintense on T2-weighted and FLAIR sequences [Figure $1 \mathrm{~b}$ and c]. Genu of CC showed iso to hypointense areas on T2weighted images which were iso to hyperintense on T1-weighted images and blooming on gradient echo sequence. Diffusion weighted imaging (DWI) and apparent diffusion coefficient (ADC) map showed restricted diffusion in the CC and bilateral hemispheric white matter and cortex [Figure 1d]. On contrast the CC lesions were seen as ring enhancing lesions and there was nodular enhancement in the left frontal lobe white matter [Figure 1e]. Fluorodeoxyglucose (FDG) PET-CT scan done 21 days after admission showed reduced cerebral glucose metabolism diffusely over the cerebral hemispheres. This reduction was accentuated in the frontal and parietal association cortices and primary sensorimotor cortices [Figure 1f]. Magnetic resonance spectroscopy (MRS) was attempted, but the signal to noise ratio was poor owing to presence of blood in the sub acute phase.

He was started on intravenous thiamine $100 \mathrm{mg}$ per day and also other vitamins. In the next two days patient improved in his sensorium but had visual hallucinations and muttering and irrelevant speech. There was gradual improvement in the sensorium over the next ten days. Neuropsychological testing revealed impaired orientation, attention and comprehension. At discharge, on day fifteen, he was doubly incontinent with no focal deficits. On telephonic interview, on June 19, 2008, it was reported that patient has joined back his work as casual laborer and is able to do his earlier work of carrying loads on to the trucks. He is self-dependent for activities of daily living. However, he continues to be dull and withdrawn with memory deficits. Further details could not be obtained. Follow-up imaging could not be done as he has refused to have the investigations done.

\section{Case 2}

A 55-year-old security guard was evaluated in July 2005. He had history of chronic alcohol abuse since 20 years and daily drinking, almost throughout the day, in the last five years. His food intake was reduced drastically. He had gradual onset bilateral intermittent hand tremor and slowness of all activities in the six months before admission. For one week prior to admission he had binge drinking of country liquor (arrack), and on the seventh day developed repeated vomiting and two days later developed behavioral abnormalities, social disinhibition, excessive irrelevant talk, memory disturbances, perseveration of speech, reduced sleep and refusal of feeds. On the third day he lapsed into altered sensorium. There was no history of seizures or focal deficits. On admission patient was unconscious and had normal papillary reaction, generalized rigidity and bilateral pyramidal signs. Laboratory investigations revealed normal hemogram was normal, liver and renal functions, serum electrolytes. Blood glucose was $70 \mathrm{mg} / \mathrm{dl}$. Lumbar CSF revealed no cells and normal biochemistry. Bacterial and fungal cultures were negative. CSF VDRL was nonreactive. Serum retroviral status was negative. Ethanol level or serum thiamine levels were not estimated. EEG showed diffuse slowing with theta and delta activity.

CT scan of brain done at admission showed diffuse hypodensity involving CC in its entire extent and also subtle frontal subcortical hypodensities [Figure 2a]. MRI showed diffuse T2 hyperintensity lesion involving the entire CC with corresponding T1-weighted hypointensity lesion. Center of the T2-weighted hyperintense lesion was markedly hypointense on T1-weighted image. There 
was a classic sandwich sign due to central necrosis of the $\mathrm{CC}$ and sparing of dorsal and ventral fibers of CC. [Figure 2b-e] Two small focal subcortical white matter T2-weighted hyperintensity lesions were noted in left parietal periventricular white matter [Figure 2c and d]. MRS of the right parietal region showed reduced
N-acetylaspartate (NAA) peak at 2.01 ppm. Choline (Ch) was mildly increased with $\mathrm{Ch} / \mathrm{Cr}$ ratio of 1.62 [Figure $2 \mathrm{f}$ ].

He was treated with intravenous thiamine $100 \mathrm{mg}$ dose per day. On the fifth day of admission patient improved minimally, had eye to eye contact and was
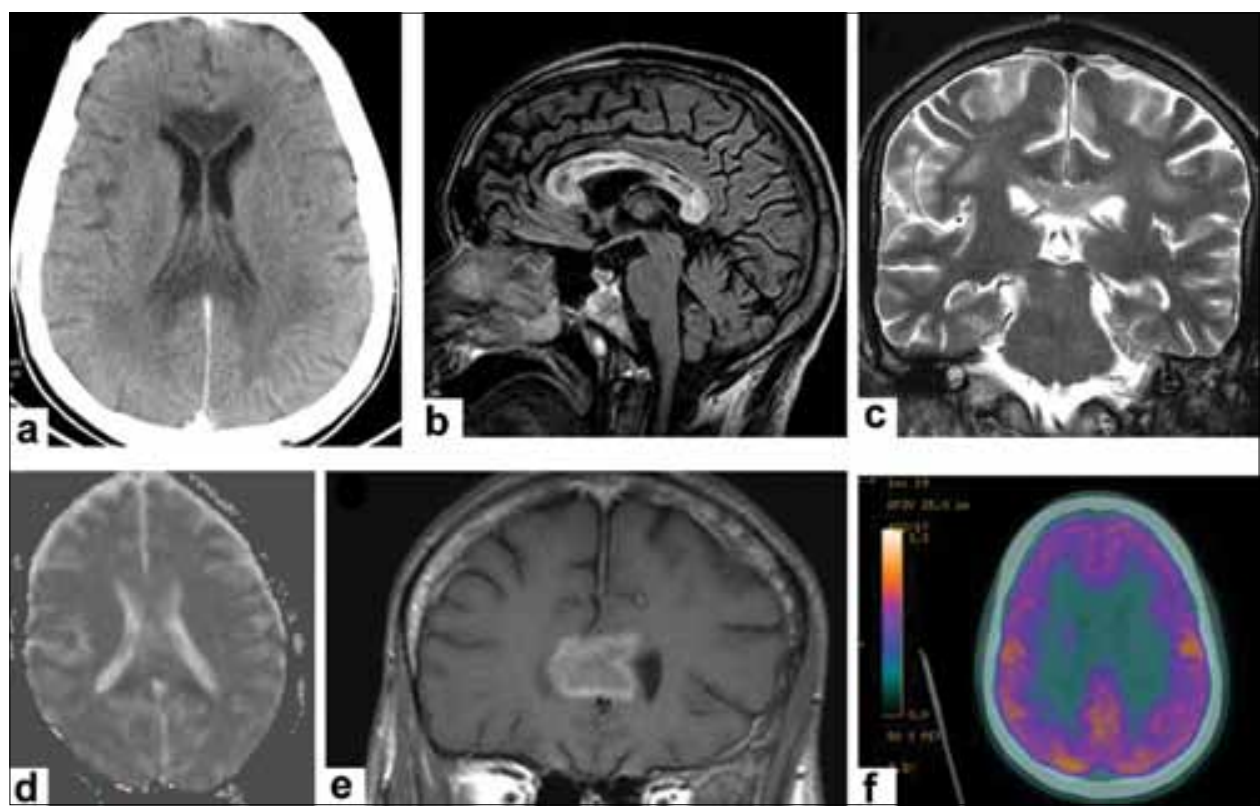

Figure 1: (a) Computer tomography brain contrast reveals diffuse hypodensity involving corpus callosum and periventricular white matter; (b) FLAIR sagg image shows peripheral hyperintensity with central hypointensity involving entire corpus callosum; (c) Coronal T2WI shows hyperintensity involving corpus callosum and bilateral hemispheric white matter; (d) Diffusion weighted imaging apparent diffusion coefficient map showing restricted diffusion in corpus callosum and bilateral hemispheric white matter and cortex; (e) Post contrast SE T1WI showing enhancing lesions in corpus callosum; (f) Positron emission tomography scan of brain showing reduced glucose metabolism in corpus callosum and bilateral hemispheric white matter
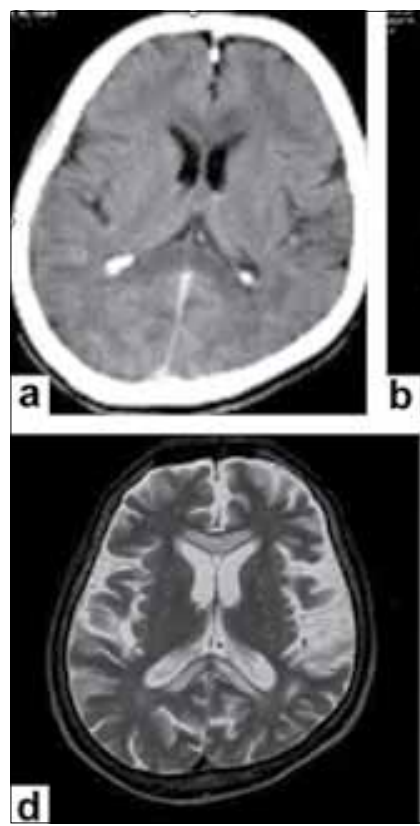
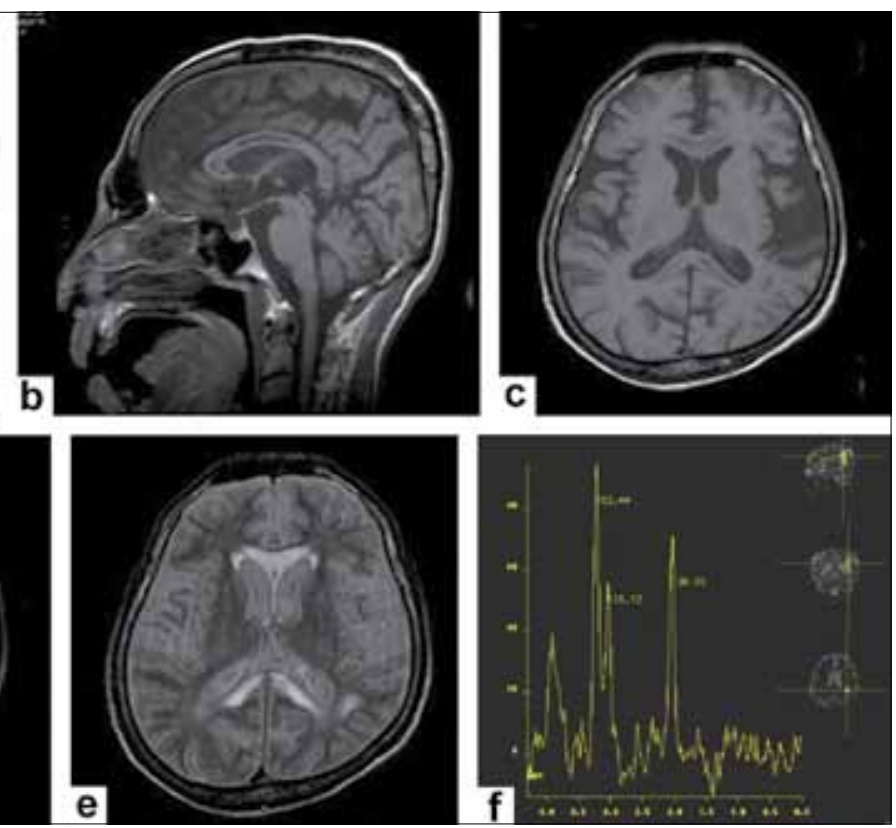

Figure 2: (a) CECT scan at level of lateral ventricle shows hypodensity of corpus callosum. The classical sandwich sign is seen; (b-c) Magnetic resonance imaging SET1WI sagg and axial images showing hypointensity involving the corpus callosum; (d) Magnetic resonance imaging SE T2WI axial image showing hyperintensity involving corpus callosum and left parietal white matter; (e) FLAIR axial image shows hyperintensity involving corpus callosum in its entire length and also left parietal white matter; (f) HMRS from left parietal lesion showing reduced N-acetylaspartate /Creatine 
in akinetic mute state and by the seventh day started verbalizing few words. He was started on levodopa and carbidopa combination for his Parkinson features. A detailed neuropsychological assessment could not be done. Findings two weeks after admission were a mute state and motor perseveration. His attention could be aroused and sustained and further testing was not possible. He came for regular follow-up and at one year he was able to speak monosyllables and could indicate personal needs. At two years he was still dependent for all activities of daily living. His last follow-up was in August 2008. He had improved significantly and had joined back work but as a gate keeper but not as security gaurd. His repeat neuropsychological assessment showed sustained attention with impaired expressive speech and could utter only a few comprehensible words. He was oriented to time, place, and person and was able to do three command step. Due to reduced verbal output and motor difficulty further assessment could not be performed. He was being continued on drugs for Parkinson's feature and repeat imaging was not performed.

\section{Discussion}

MBD was first described by Carducci in 1898 in Italian red wine drinkers and by Marchiafava and Bignami in 1903..$^{[12,13]}$ This syndrome occurs predominantly in malnourished alcoholics and reported more in men than women drinkers. ${ }^{[14,15]}$ It has been reported that patients who consume at least 2 litres of red wine for more than 20 years are likely to suffer from MBD. ${ }^{[16]}$ Our patients had harmful use of alcohol for four to five years only and present with acute form of MBD. MBD has been rarely reported in non-alcoholic malnourished patients, thus suggesting that alcohol per se has a causative relation with MBD. ${ }^{[17]}$ MR imaging is the most appropriate investigative modality to evaluate the CNS changes in MBD. The distinctive characteristics of MBD are selective demyelination of CC with or without necrosis. T2 prolongation on MRI is due in part to edema in addition to myelin damage and in acute phase CC is hyperintense. ${ }^{[18]}$ The CC may remain hyperintense when there is permanent damage to the myeline and with complete remyelination the CC signals may return to normal. ${ }^{[18]}$ In both the patients $\mathrm{CC}$ was hyperintense on T2-weighted sequences. Necrosis and cyst formation can occur especially in the genu and splenium. ${ }^{[19,20]}$ Both our patients had necrotic lesions in the CC and in of the patients the lesions demonstrated ring enhancement. Bleeding can occur in the subacute phase, ${ }^{[9,21]}$ and the hypointensity on T2-weighted images correlates with hemosiderin deposit. ${ }^{[22]}$ This finding was observed in our first patient. Focal hyperintensities have been reported in periventricular white matter which are non-specific and probably represent the extension of damage. ${ }^{[3]}$ Our patients had hyperintensities in the corona radiata and periventricular white matter.

The presenting features in MBD are non-specific and non-diagnostic. The course of the disease may be acute, subacute or chronic and may lead to death within weeks or months. ${ }^{[23]}$ Hemispherical disconnection syndromes may be seen in patients who survive the disease ${ }^{[2]}$ Both our patients presented with alterations in mental status and had the characteristic CT and MRI features. They both survived the acute phase of the illness. The first patient at two and half months follow-up after discharge has been able to go back to his previous occupation and the second patient could be rehabilitated in a less demanding job in the same work place.

Pappata et al., ${ }^{[10]}$ demonstrated markedly reduced glucose metabolism on PET studies in the frontal and temporo-parieto-occipital association cortices in a patient with alcohol-related MBD. ${ }^{[10]}$ PET-CT study in our first patient demonstrated reduced cerebral glucose metabolism over both the cerebral hemispheres, more so in the frontal and parietal association cortices and primary sensorimotor cortices. In our patient, glucose metabolism was reduced markedly where as MRI showed lesions essentially limited to corpus callosum and white matter. Similar were the observations in the other studies also. ${ }^{[10,11]}$ These observations may suggest that in MBD perfusion and metabolism defects may affect structures beyond the CC like cerebral hemispheres and the widespread cerebral functional involvement may account for the complex neurologic and cognitive deficits. The cognitive deficits probably may be related to the functional abnormalties of the frontal and parietal lobes. The findings suggest that disconnection of not only commissural fibers but also the extracallosal projection and association fibers involving the cortico-cortical and cortico-subcortical connectivity can cause transneural depression of the cortical metabolism and function. Single photon emission computed tomography performed on a patient with MBD did not demonstrate areas of focal hypoperfusion, however $\mathrm{CT}$ scan and MRI showed scattered lesions in the CC and extensive symmetrical lesions in both the centrum semiovale. ${ }^{[11,24]}$

Serial studies of MRI and MRS in a patient with MBD who had extensive signal abnormalities in the CC at $1,2,4$, and 11 months showed progressive reduction in signal intensity abnormalities and residual callosal atrophy. MRS showed progressive reduction in the NAA: Cr ratio with partial reduction in the last study and normalization of the choline: $\mathrm{Cr}$ ratio, which was initially slightly increased. ${ }^{[25]}$ MRS in our second patient demonstrated reduced NAA/creatine ratio suggestive of neuronal loss secondary to demyelination. 
Till recently the diagnostic confirmation of MBD was at autopsy. However, in the present era the diagnosis of MBD can fairly be established antimartum by MRI imaging in a given clinical setting. ${ }^{[18]}$ Our two patients illustrate that early diagnosis and early institution of appropriate treatment may be associated with reasonable good outcomes.

\section{References}

1. Castaigne P, Buge A, Cambier J, Escourolle R, Rancurel G. La Maladie de Marchiafava-Bignami: Etude anatomoclinique de dix observatios. Rev Neurol 1971;125:179-96.

2. Rosa A, Demiati M, Cartz L, Mizon JP. Marchiafava-Bignami disease, syndrome of interhemispheric disconnection, and right-handed agraphia in a left-hander. Arch Neurol 1991;48:986-8.

3. Izquierdo G, Quesada MA, Chacon J, Martel J. Neuroradiologic abnormalities in Marchiafava-Bignami disease of benignevolution. Eur J Radiol 1992;15:71-4.

4. Nicoli F, Vion-Dury J Chave B, Houallah T, Confort-Gouny S, Cozzone PJ, et al. Maladie de Marchiafav-Bignami:Disconnexion interhemispherique, syndrome de Balint, evolution spontanement favorable. Rev Neurol 1994;150:158-61.

5. Shiota J, Kawamura M, Hirayama K, Isono O, Maki T. Antemortem diagnosis of Marchiafava-Bignami disease. Rinsho Shinkeigaku 1989;29:701-6

6. Lechevalier B, Anderson JC. Les troubles du transfert interhemispheriques. Rapport de Neurologie du congres de psychictrie et Neurologie de Langue Fancaise, $73^{\circ}$ Session, Nimes, Paris, Masson: 1975. p. 163-4 and 173-4.

7. Valk J, van der Knaap MS. Marchiafava-Bignami syndrome. In: Valk J, van der Knaap MS, editors. Magnetic resonance of myelin, myelination and myelin disorders. Berlin: Springer-Verlag; 1989. p. 265-7.

8. Berek K, Wagner M, Chemelli AP, Aichner F, Benke T. Hemispheric disconnection in Marchiafava-Bignami disease: Clinical, neuropsychological and MRI findings. J Neurol Sci 1994;123:2-5.

9. Kamaki M, Kawamura M, Moriya H, Hirayama K. Callosal bleeding in a case of Marchiafava-Bignami disease. J Neurol Sci 1996;136:86-9.

10. Pappata S, Chabriat H, Levasseur M, Legault-Demare F, Baron JC. Marchiafava-Bignami disease with dementia: Severe cerebral metabolic depression revealed by PET. J Neural Transm Park Dis Dement Sect 1994;8:131-7.

11. Ferracei F, Conte F, Gentile M, Candeago R, Foscolo L, Bendini M.
Marchiafava-Bignami disease: Computed tomographic scan, $99 \mathrm{mTc}$ HMPAO-SPECT, and FLAIR MRI findings in a patient with subcortical aphasia, alexia, bilateral agraphia, and left-handed deficit of constructional ability. Arch Neurol 1999;56:107-10.

12. Carducci A. Contributo allo studio delle encefaliti non suppurate. Riv Psicol Psichiat Neuropat 1898;8:125-35.

13. Marchiafava E, Bignami A. Sopra un alterzione del corpo calloso osservata in soggetti alcoolisti. Riv Pat Nerv Ment 1903;8:544-9.

14. Harper C, Butterworth R. Nutritional and metabolic disorders. In: Graham DI, Lantos PL, editors. Greenfield's Neuropathology. $6^{\text {th }}$ ed. London: Arnold; 1997. p. 616-7.

15. Kohler CG, Ances BM, Coleman AR, Ragland JD, Lazarev M, Gur RC. Marchiafava-Bignami disease. Neurology 2000;13:67-76.

16. Brion S. Marchiafava-Bignami disease. In: Vinken PJ, Bruyn GW, editors. Handbook of clinical neurology. Amsterdam: North H: 1977. p. 317-29.

17. Rickert CH, Karger B, Varchmin-Schultheiss K, Brinkmann B, Paulus W. Neglect-associated fatal Marchiafava-Bignami disease in a non-alcoholic woman. Int J Legal Med 2001;115:90-3.

18. Bracard S, Claude D, Vespifnani H, Almeras M, Carsin M, Lambert H. Computerized tomography and MRI in Marchiafava-Bignami disease. J Neuroradiology 1986;13:87-94.

19. Ghatak NR, Hadfield MG, Rosenblum WI. Association of central pontine myelinolysis and Marchiafave-Bignami disease. Neurology 1978;28:1295-8.

20. Koeppen AH, Barron KD. Marchiafava-Bignami disease. Neurology 1978;28:290-4

21. Chang KH, Cha SH, Han MH. Marchiafava-Bignami disease: Serial changes in corpus callosum on MRI. Neuroradiology 1992;34:480-2.

22. Baron R, Heuser K, Marioth G. Marchaifava-Bignami disease with recovery diagnosed by CT and MRI: Demyelination affects several CNS structures. J Neurol 1989;236:364-6.

23. Adams RD, Victor M, Ropper AH. Disease of the nervous system due to nutrinional deficiency. In: Principles of Neurology. New York: McGraw-Hill; 1997. p. 1158-9.

24. Humbert T, De Guilhermier P, Maktouf G, Grasset G, Lopez FM, Chabrand P. Marchiafava-Bignami disease: A case studied by structural and functional imaging. Eur Arch Psychiat Clin Neuroscience 2005;242:69-71.

25. Gambini A, Falini A, Moiola L, Comi G, Scotti G. Marchiafava-Bignami disease: Longitudinal MR imaging and MR spectroscopy study. AJNR $2003 ; 24: 249-53$.

Accepted on 02-07-2009

Source of Support: Nil, Conflict of Interest: None declared. 